\title{
Class-A Operation of InAs Quantum Dash-based Vertical- External-Cavity Surface-Emitting Laser
}

\author{
Salvatore Pes ${ }^{1,2}$, Kevin Audo ${ }^{2}$, Cyril Paranthoën ${ }^{1}$, Christophe Levallois ${ }^{1}$, Nicolas Chevalier ${ }^{1}$, Goulc'hen Loas ${ }^{2}$, \\ Steve Bouhier ${ }^{2}$, Cyril Hamel ${ }^{2}$, Carmen Gomez ${ }^{3}$, Jean-Christophe Harmand ${ }^{3}$, Sophie Bouchoule ${ }^{3}$, Hervé \\ Folliot $^{1}$, Mehdi Alouini ${ }^{2}$ \\ ${ }^{1}$ FOTON, UMR CNRS 6082, INSA de Rennes, 35708 Rennes, France \\ ${ }^{2}$ Institut de Physique de Rennes, UMR UR1-CNRS 6251, Université de Rennes 1, 35042 Rennes, France \\ ${ }^{3}$ Centre de Nanosciences et de Nanotechnologies, CNRS, Université Paris-Sud, 91360 Marcoussis, France \\ salvatore.pes@insa-rennes.fr
}

\begin{abstract}
InAs Quantum Dash-based Vertical-External-Cavity Surface-Emitting Laser on InP is demonstrated. Up to $163 \mathrm{~mW}$ and $7 \mathrm{~mW}$ have been obtained in multi-mode and single-mode operation, respectively. Class-A behavior is demonstrated on such device.

OCIS codes: 140.0140 Lasers and laser optics; 140.5960 Semiconductor lasers; 140.7260 Vertical cavity surface emitting lasers; 250.5590 Quantum-well, -wire and -dot devices; 350.4010 Microwaves.
\end{abstract}

Since their first demonstration in 1997 [1], Vertical-External-Cavity Surface-Emitting Lasers (VECSELs, also known as Semiconductor Disk Lasers, SDLs) have gained much attention. They can be used in numerous applications, ranging from optical sensing, frequency doubling, ultrafast photonics, high resolution spectroscopy and microwave photonics. Beside their high power and good quality circular beam, VECSELs offer the advantage to use intra-cavity optical elements (non-linear crystals, saturable absorbers, etalons, etc.) to further extend their functionalities. Moreover, VECSELs have been demonstrated to operate in the so-called Class-A regime (free of relaxation-oscillations phenomena), provided that the cavity is long enough [2]. In such a regime, VECSELs show extremely low Relative Intensity Noise (RIN) levels, down to the shot-noise. On the other hand, quantum dashbased active media have been demonstrated to ensure a stable state of polarization of the emitted light $[3,4]$, together with a wide material gain [5] and a lower sensitivity to temperature. The original integration of such an active region into a VECSEL configuration is thus expected to extend existing functionalities and to open the way to new ones.

Here we present preliminary results on an optically-pumped InAs quantum dash-based VECSEL (OP-QDHVECSEL) on a (001)-oriented InP substrate, emitting in the telecom L-band (around $1619 \mathrm{~nm}$ ). In the following, the laser structure is described. The multi-mode output power characteristic and optical spectrum are presented. Class-A operation of the laser is finally demonstrated. To the best of our knowledge, this is the first experimental demonstration of a QDH-VECSEL on InP.
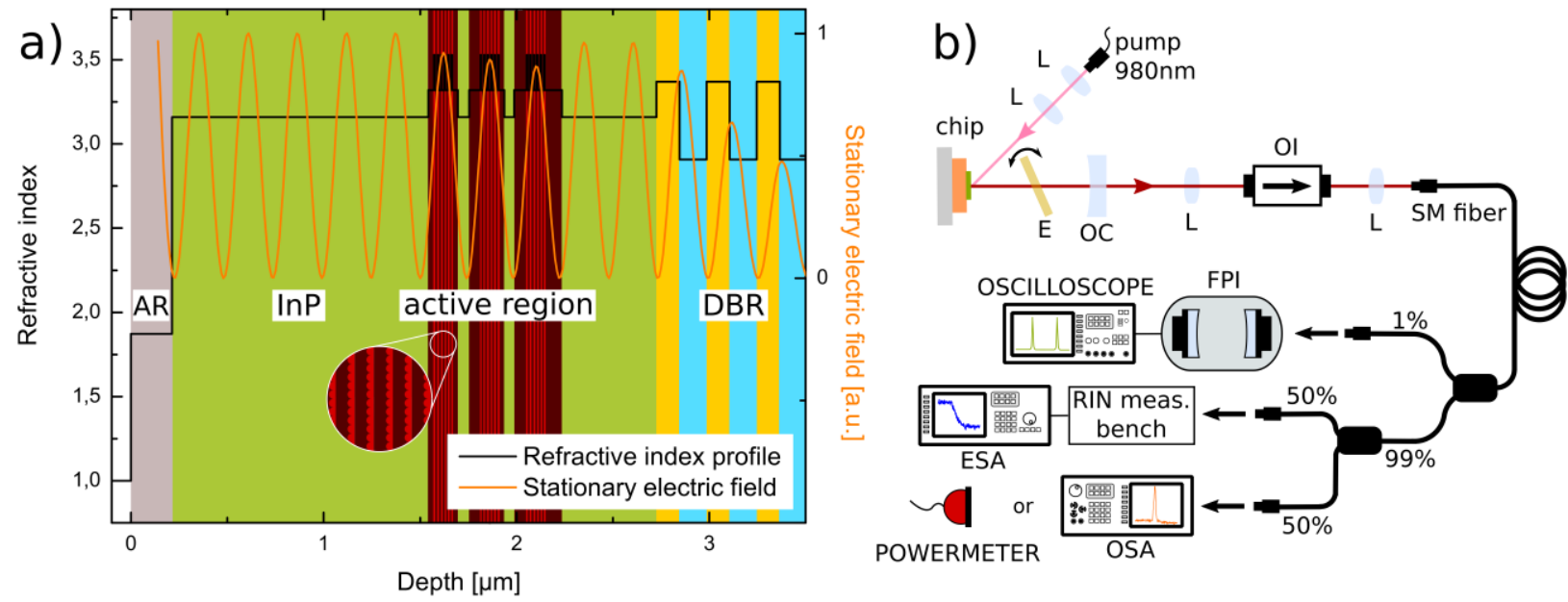

Fig. 1: a) OP-QDH-VECSEL design and computed stationary electric field inside the structure; b) experimental setup. L: lens, E:etalon, OC: output coupler, OI: optical isolator, FPI: Fabry-Perot interferometer, ESA/OSA: electrical/optical spectrum analyzer.

Fig. 1a shows the detailed structure of the QDH-VECSEL. The active region has been grown by a gas-source MBE system on a (001)-oriented InP substrate. It consists of 3 sets of 6 planes of self-assembled InAs quantum dashes placed on the antinodes of the stationary electric field (Resonant Periodic Gain configuration). Each QDH 
plane is surrounded by $\mathrm{Ga}_{0.2} \mathrm{In}_{0.8} \mathrm{As}_{0.435} \mathrm{P}_{0.565}$ barriers to ensure the carrier confinement and to enhance the pump absorption. The bottom Distributed Bragg Reflector (DBR) consists in 17 periods of $\mathrm{GaAs} / \mathrm{Al}_{0.97} \mathrm{Ga}_{0.03} \mathrm{As}_{\text {layers, }}$, which has been metamorphically grown on the InP-based structure, completed with a gold metallic layer (not shown in the figure). After metallic bonding onto a CVD diamond host substrate, the InP substrate has been removed and the whole structure has been fixed to a temperature-controlled copper thermal sink [6]. A $\mathrm{SiN}_{\mathrm{x}}$ anti-reflection coating at the emission wavelength has been added on the top surface.

The sample has been tested in the cavity set-up shown in Fig. 1b. The output power characteristic and the spectrum presented in Fig. 2a were obtained using a multi-mode $980 \mathrm{~nm}$ semiconductor laser pump with a $145 \mu \mathrm{m} \mathrm{x}$ $210 \mu \mathrm{m}$ spot, and the QDH-VECSEL was operating in the multi-mode regime. The cavity length was set to $12 \mathrm{~mm}$ and no etalon was inserted inside the laser cavity. Under these conditions, a fundamental mode diameter of $\sim 120 \mu \mathrm{m}$ on the chip surface was estimated. Up to $163 \mathrm{~mW}$ output power at $\mathrm{T}=20^{\circ} \mathrm{C}$ has been obtained for an incident power of $7 \mathrm{~W}$ (pump limited), with an emission centered at $1619 \mathrm{~nm}$.
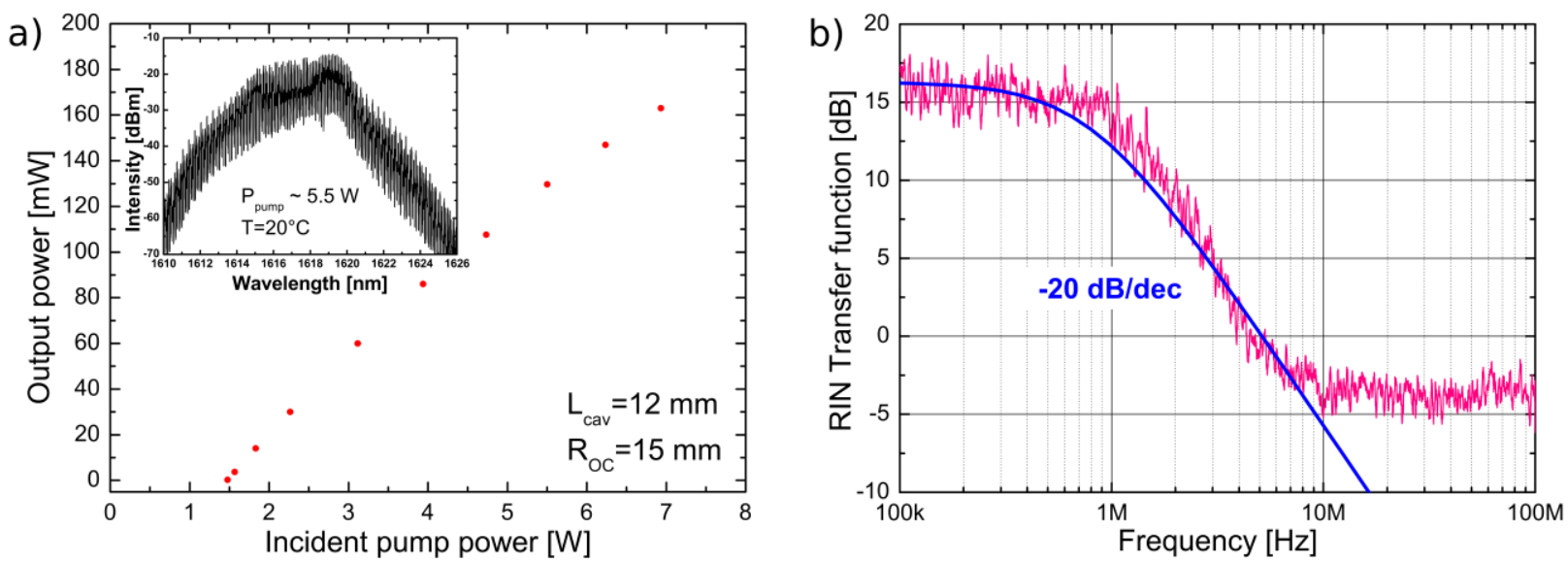

Fig. 2: a) output power characteristic and optical spectrum (inset) of the QDH-VECSEL in multi-mode operation $\left(\mathrm{T}=20^{\circ} \mathrm{C}\right.$, radius of curvature of the output coupler is $15 \mathrm{~mm}$ ). b) RIN transfer function of the laser (obtained for $\mathrm{I}_{\mathrm{ph}}=2 \mathrm{~mA}$ ).

In order to obtain the Class-A regime for the QDH-VECSEL, we optimized the setup in terms of noise by using a single-mode semiconductor pump laser ( $980 \mathrm{~nm}$, pump spot of $80 \mu \mathrm{m} \times 120 \mu \mathrm{m}$ ), extending the laser cavity up to $49 \mathrm{~mm}\left(\mathrm{R}_{\mathrm{OC}}=50 \mathrm{~mm}\right)$ and adding a $40 \mu \mathrm{m}$ thick fused silica etalon with $\mathrm{R}=30 \%$ on both faces, to ensure the longitudinal single-mode operation of the QDH-VECSEL (checked with a Fabry-Perot interferometer). In such conditions, the laser emits $7 \mathrm{~mW}$ at $1610 \mathrm{~nm}$ when pumped at $1 \mathrm{~W}$. We acquired the RIN spectra of both QDHVECSEL and pump with a dedicated RIN measurement bench composed by a InGaAs fast photodiode, two RF amplifiers and an ESA. A shot-noise level value of $-158 \mathrm{~dB} / \mathrm{Hz}$ has been obtained for our QDH-VECSEL, for a detected photocurrent of $2 \mathrm{~mA}$. The RIN transfer function, after pump normalization, is given in Fig. $2 \mathrm{~b}$ : it shows that the QDH-VECSEL acts like a 1st order filter with a cut-off frequency of about $800 \mathrm{kHz}$ and a slope of -20 $\mathrm{dB} / \mathrm{dec}$, which confirms the Class-A behavior.

Further characterization are underway, in order to investigate quantum dash-based active medium properties in low-noise external cavity configuration.

Acknowledgement: This work was supported by French agencies ANR (Agence Nationale de la Recherche) and DGA (Direction Générale de l'Armement) within the ANR-ASTRID HYPOCAMP project (Grant n ${ }^{\circ}$ ANR-14-ASTR-0007-01) and a DGA-MRIS and Brittany Region scholarship (Grant ARED-VELOCE n8917 - Région Bretagne).

[1] M. Kuznetsov et al., "High-power (>0.5-W CW) diode-pumped vertical-external-cavity surface-emitting semiconductor lasers with circular TEM $_{00}$ beams", IEEE Photonics Technology Letters 9, 1063-1065 (1997).

[2] G. Baili et al., "Shot-noise-limited operation of a monomode high-cavity-finesse semiconductor laser for microwave photonics applications", Optics Letters 32, 650-652 (2007).

[3] J. M. Lamy et al., "Polarization control of $1.6 \mu \mathrm{m}$ vertical-cavity surface-emitting lasers using InAs quantum dashes on InP(001)", Applied Physic Letters 95, 011117 (2009).

[4] J.-P. Gauthier et al., "Enhancement of the polarization stability of a $1.55 \mu \mathrm{m}$ emitting Vertical-Cavity Surface-Emitting Laser under modulation using quantum dashes", Optics Express 20, 16832-16837 (2012).

[5] F. Taleb et al., "VCSEL Based on InAs Quantum-Dashes With a Lasing Operation Over a 117-nm Wavelength Span", IEEE Photonics Technology Letters 25, 2126-2128 (2013).

[6] Z. Zhao et al., "Subpicosecond pulse generation from a 1.56 $\mu$ m mode-locked VECSEL", Optics Letters 36, 4377-4379 (2011). 